\title{
Theory of computation: non-P systems-Articles dedicated to Kamala Krithivasan on her 70th birthday, issue-2
}

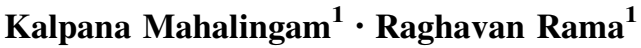

Published online: 10 April 2019

(C) Indian Institute of Technology Madras 2019

This is the second issue in honour of Prof. Kamala Krithivasan on her 70th birthday, which comprises of contributions invited by the guest editors.

With a striking difference from the first issue which concentrates on $\mathrm{P}$ systems, the current issue has incorporated a wide range of contents on recent developments in other areas of theoretical computer science. The precise sequencing and presentation are well managed in seven articles. The first two articles fall under the category of Automata and languages. The first article studies the similarity between any two given tree automata using the concept of edit distance while the second discusses the generative power of matrix insertion-deletion systems.
Some popular combinatorial problems restricted to special types of graphs are studied in papers 3 and 4 . The chemical compounds that comprise of atoms and bonds are conceptualized as graphs, and their properties are studied using M-polynomials in paper 5. Paper 6 deals with a discrete minimization problem of bounded depth $(\mathrm{min} ;+)$ formulas computing the shortest path polynomial. The last of this issue, on data science, provides a clustering algorithm to detect outliers in high-dimensional data.

The guest editors take this opportunity to thank the authors for contributing to these special issues, the referees for their timely and critical reviews, and the Springer publishing team for their cooperation.

Kalpana Mahalingam

kmahalingam@iitm.ac.in

1 Department of Mathematics, Indian Institute of Technology, Chennai, India 